\title{
Rezension
}

András Wienands (Hrsg.): System und Körper. Der Körper als Ressource in der systemischen Therapie. Vandenhoeck \& Ruprecht, Göttingen, 2014, 245 Seiten, $€ 24,99$ (D)

System und Körper - da fragen sich vermutlich einige Menschen, egal, ob Fachkräfte oder einfach Interessierte an der Thematik -, wie passt denn das zusammen bzw. was haben der Körper oder der Einsatz des Körpers mit dem "System « oder dem systemischen Arbeiten zu tun? Als klare Antwort kann hier inzwischen folgen: eine ganze Menge. Körper(arbeit) hat in therapeutischen Prozessen schon lange Einzug gehalten und der Nutzen daraus wird wissenschaftlich erforscht.

Auch wenn die systemische Praxis methodisch sehr breit aufgestellt ist oder gerade deshalb - nutzen immer mehr Fachkräfte die Wirkung des Körpers bzw. der Körperarbeit in der systemischen Praxis. Sie erheben sich bildlich gesprochen aus der »klassischen Liegestuhlposition « der Therapeuten und kommen mit den Klienten in Bewegung.

András Wienands hat es sich als Herausgeber zur Aufgabe gemacht, ein Buch zu erschaffen, das verschiedene Einsatzmöglichkeiten des Körpers als Ressource in Therapie, Beratung und Coaching herausfiltert. Hier ist von Bedeutung, dass er sich nicht nur auf die Arbeit mit Familien beruft, sondern alle vier relevanten systemischen Arbeitsbereiche (Familie, Paare, Einzelne und Coaching) gleichermaßen berücksichtigt. Anhand von vierzehn Beispie- len, eingebettet in diese vier Arbeitsbereiche, zeigen die AutorInnen sehr anschaulich, welche Ressource der Körper in der systemischen Arbeit darstellt. Nach einer kurzen Einleitung erfolgt eine Zusammenfassung von vierzehn Therapiesettings innerhalb dieser Arbeitsbereiche, die von den jeweiligen Autorlnnen kurz und prägnant vorgestellt werden. Jedes Beispiel beginnt mit einer Einleitung gefolgt von einem Fallbeispiel oder der Beschreibung des methodischen Vorgehens sowie Übungen, durch welche die entsprechende Arbeitsweise nachvollziehbar wird. Die Fallbeispiele verdeutlichen den Leserlnnen, wie der Körper als Ressource in das jeweilige Konzept integriert werden kann. Besonders interessant sind neben den Fallbeispielen auch die Vorstellung verschiedener Interventionsformen, aber auch Übungen aus der therapeutischen Praxis, die sehr nachvollziehbar die therapeutische Vorgehensweise darlegen.

Im Arbeitsbereich Familie wird der Körper als Ressource in der systemischen Therapie mit Familien und Kindern durch die Therapieansätze von drei Autoren vorgestellt. Aichinger hebt in seinem Beitrag »Mit Familien spielen « das psychodramatische Familienspiel hervor, das einerseits den Kindern ermöglicht, verlorengegangene Bereiche des Körpers wiederzuentde-

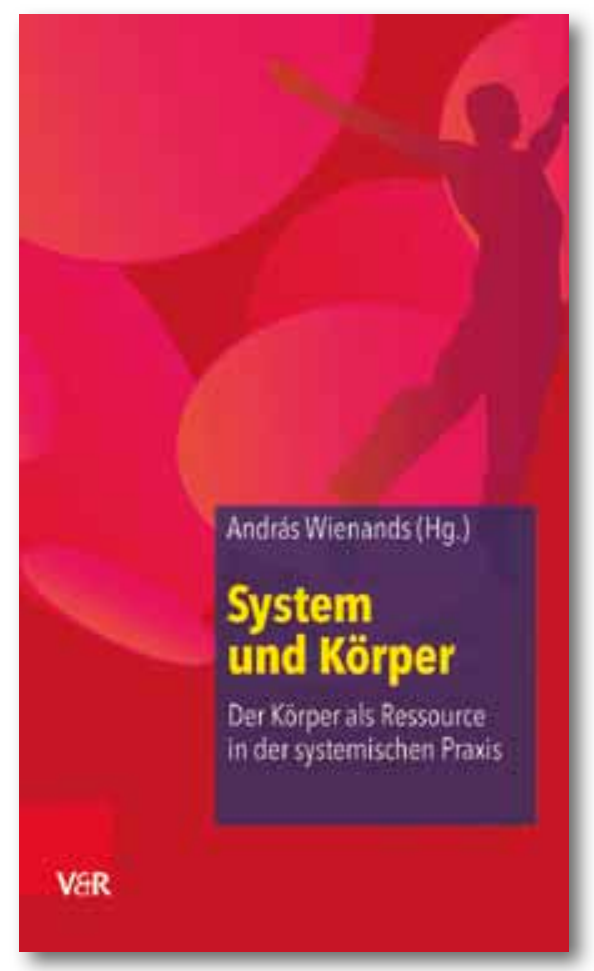

cken und einen Zugang zur Selbststeuerung zurückzugewinnen sowie anderseits den Eltern verhilft, ihre Kinder entwicklungsfördernd $\mathrm{zu}$ unterstützen. In dem Beitrag von Kachler wird die "Trauerarbeit als hypno-systemische Körperarbeit« vorgestellt. Diese soll den Betroffenen aufzeigen, wie sie ihr Leben mit einem verstorbenen Menschen gestalten können. Hier dient der Körper als Ressource zur Bewältigung und Neugestaltung. Der Ansatz von Richter-Mackenstein beschäftigt sich mit der systemisch-psychomotorischen Familienberatung. Systemische Beratungs- / Therapieprozesse werden durch psychomotorische Elemente unterstützt. Das Spiel steht im Vordergrund und die daraus sich entwickelnden Interaktionen werden an- 
schließend therapeutisch aufgearbeitet.

Im Arbeitsbereich Paare wird »der Körper als Ressource in der systemischen Therapie mit Paaren « thematisiert. Hier gelingt es in drei Beiträgen, Paaren die Möglichkeit zu verschaffen, die Routinen des Alltags zu durchbrechen und ihrem Paar- und Sexualleben wieder mehr Aufmerksamkeit zu widmen. Günther ermöglicht dies durch sein Konzept der systemischen Sexualtherapie. Stephan / El Hachimi stellen z.B. durch Achtsamkeitsübungen vor, wie sich Paare wieder näher kommen. Beier / Lütteke beschreiben die tiefere Verbindung zwischen den PartnerInnen für eine Annäherung und Auseinandersetzung.

Die systemische Therapie mit Einzelnen wird durch fünf Autorlnnen vorgestellt. Wienands befasst sich in seinem Beitrag »Körperorientierte systemische Therapie«, damit, wie der Körper / Körperarbeit nutzbar gemacht werden, um u.a. Gefühle oder Bewegungen zuzulassen, die als eher problematisch erlebt wurden. Korittko beschäftigt sich mit dem Thema Trauma und Körper. Hier werden sehr ansprechend erste Lösungsschritte einer Körper-, Ressourcen- und Systemorientierten Traumatherapie vorstellt. Strübing / Roming nutzen in ihrer Arbeitsweise eine Technik aus dem Psychodrama - das Doppeln -, um durch die Arbeit mit dem Körper als lähmend empfundene Situationen bearbeiten zu können. Ein weiterer Ansatz, der für die Arbeit mit Einzelnen vorgestellt wird, ist die Im- protherapie im Beitrag von Dshemuchadse / Scherbaum, die Teile aus dem Improtheater und der systemischen Therapie miteinander verknüpft. BreitSchröder indessen beschreibt körperorientierte Interventionen in der systemischen Therapie, die auf Konzepten von George Downing beruhen. Hier werden frühkindliche Beziehungserfahrungen und ihre Auswirkungen auf das Erwachsenenalter vorgestellt.

Der Arbeitskontext Coaching wird durch drei Autorlnnen vorgestellt, die den Körper als Ressource im systemischen Coaching nutzen. Bohmann / Bohmann zeigen auf, wie der Einsatz des Körpers als »Türöffner « und die damit verbundenen körperlichen Signale zu Erkenntnisgewinn und Lösungen führen können. Ahrendt stellt das Konzept »Kommunikation mit dem Körper mittels Kinesiologie« vor, in dem Körpersignale des Coaches zur Ressourcenaktivierung im Vordergrund stehen. Zu guter Letzt erläutert Amann die Arbeitsweise »Resilienzförderung nach dem Bambus-Prinzip«, das Teile aus der Improvisation und dem BodyblissTraining nach Müller beinhaltet.

Alle Beiträge beinhalten eine einleitende Basis, indem zunächst die theoretische Einbettung des Ansatzes vorgenommen wird. Um ein besseres Verständnis für die Theorie zu erhalten, werden Fallbeispiele, Interventionen, Lösungsschritte, je nach Beitrag, eingebettet.

Zusammenfassend kann festgehalten werden: Das vorliegende Buch liefert eine Vielzahl von detaillierten, auf aktueller Forschung beruhenden Therapieansätzen, die den Körper in verschiedenen Settings als Ressource in den systemischen Kontext einbeziehen.

Wienands als Diplom-Psychologe und Systemischer Berater/Therapeut hat mit diesem Buch ein Werk zusam mengestellt, das Menschen, die an diesem Bereich interessiert und in ihm tätig sind, hilft, einen Überblick über eine Vielzahl von Einsatzmöglichkeiten zu erhalten. Das Buch animiert durch die ausgewählten Fallbeispiele, methodischen Bausteine und Übungen dazu, in der eigenen Arbeit in Bewegung zu kommen. Inwieweit die LeserInnen die Ausführungen in ihre Praxis integrieren können, hängt sicherlich von ihrer Profession ab. Themengebiete, die Interessierten noch nicht vertraut sind, werden durch die theoretische Verknüpfung mit der gängigen Praxis anschaulich dargestellt.

Das vorliegende Buch ist in einer flüssigen Lesart geschrieben und zeigt einen gut strukturierten Aufbau. Insgesamt ein sehr gelungenes und empfehlenswertes Werk, das durch die Vorstellung der unterschiedlichen Themenbereiche PädagogInnen, PsychologInnen, Systemische Beratelnnen / TherapeutInnen, die an der Thematik interessiert sind, gleichermaßen anspricht und das Interesse weckt, die eigene Praxis zu überprüfen und Neues auszuprobieren.

\section{Yvonne Borggräfe}

DOI 10.2378 / motorik2015.art15d 